\title{
Respiratory Mucosal Edema Alteration
}

National Cancer Institute

\section{Source}

National Cancer Institute. Respiratory Mucosal Edema Alteration. NCI Thesaurus. Code C40940.

Respiratory Mucosal Edema Alteration involves a change in the quality of the existing state of swelling in the respiratory tract mucosa due to abnormally large amounts of fluid in the extracellular space. Such alterations may increase or decrease blockage of airways. 